\title{
Editorial
}

\section{Re-evaluating the Optimal Exercise for the Critical Peri and Postmenopausal Years}

\author{
Ifigeneia Giannopoulou, PhD* \\ Senior Lecturer in Sport and Exercise Science, School of Sport and Service Management, University of Brighton, Eastbourne, Brighton20 7SR, UK \\ ${ }^{*}$ Corresponding author \\ Ifigeneia Giannopoulou, PhD \\ Senior Lecturer in Sport and Exercise Science, School of Sport and Service Management, University of Brighton, Eastbourne, Brighton20 7SR, UK; \\ Tel.0127364373I; E-mail: I.Giannopoulou@brighton.ac.uk
}

Article information

Received: March 7 $7^{\text {th }}, 2019$; Accepted: March 25 $5^{\text {th }}$, 2019; Published: March 28 ${ }^{\text {th }}, 2019$

\section{Cite this article}

Giannopoulou I. Re-evaluating the optimal exercise for the critical peri and postmenopausal years. Obes Res Open J. 20I9; 6(I): el-e4. doi: I0.I7I40/OROJ-6-e0I4

$\mathrm{M}$ enopause is characterized by marked changes in the circulating estrogen of the female body. ${ }^{1}$ As women age, progressively declines in ovarian function lead to a gradual reduction in estrogen secretion that ultimately ceases when menopause is reached. This transitional time is termed the perimenopausal period and it occurs approximately within one year of its commencement. ${ }^{2}$ The perimenopausal years are crucial for the future health of females as the observed hormonal alterations can lead to a number of psychophysiological changes that can increase the risk of disease if not prevented early. ${ }^{2}$ Specifically, during the perimenopausal years the diminished concentrations of estrogens, reduce their protective role on health and contribute to significant physiological disturbances. ${ }^{3}$ Adverse health effects such as reductions in fat-free mass (sarcopenia), accumulation of body fat and intra-abdominal visceral fat and associated adverse cardiovascular and metabolic impairments namely hypertension, atherosclerosis and insulin resistance are observed. ${ }^{1}$ Consequently, there is a significantly increased risk in the development of obesity and its comorbidities namely cardiovascular disease and type 2 diabetes, that can reduce the quality of life and dramatically increase the risk of mortality in older women. ${ }^{3}$ In conjunction with the aforementioned conditions, significant impairments in cognitive and mood state are observed in the peri and postmenopausal years, further increasing the risk of morbidity and mortality in older women. ${ }^{4-6}$ An increased prevalence of anxiety and depression and cognitive declines such as loss of concentration, memory and a high risk of developing dementia and Alzheimer's disease have been noted, recognizing menopause not only as a reproductive but also as a neurological transitional state. $^{7-9}$ These effects are exacerbated by the declines in physical activity participation and low exercise compliance observed in older women. The complex and multifaceted pathophysiology of the peri and postmenopausal years, makes the treatment of the aforementioned comorbidities very difficult and challenging and warrants further research on identifying the ideal treatment plan for this population. Particularly, preventive treatment plans during the perimenopausal years are deemed essential in slowing down or delaying the development of chronic mental and non-mental diseases later in life and increasing the well-being and quality of life of older women.

It is well established that exercise is an essential treatment plan for the prevention of chronic diseases in older women. ${ }^{10} \mathrm{~A}$ number of research studies have demonstrated the central role that regular endurance exercise has on the health, well-being, and quality of life of older women by partly attenuating some of the physiological changes occurring with aging. ${ }^{11,12}$ In healthy postmenopausal women, participation in moderate intensity continuous exercise training alone has been shown to induce improvements in body fat and abdominal visceral adiposity, insulin resistance, lipid levels and inflammatory markers that can be further accelerated when exercise is combined with a balanced nutrition plan. ${ }^{13,14}$ In overweight and obese postmenopausal women, regular moderate-intensity endurance exercise for 3-4 months has been well documented to improve body mass index, body composition and insulin sensitivity. ${ }^{15}$ In type 2 diabetic obese postmenopausal women, moderate intensity endurance exercise can induce significant improvements in glycemic control, Hemoglobin A1c and inflammatory markers primarily due to reductions in intra-abdominal visceral adiposity and increases in exercise capacity. ${ }^{16-18}$ While, in hypertensive postmenopausal women there is strong evidence to support the important role of endurance exercise on reducing cardiovascular risk factors through significant improvements in blood pressure, autonomic tone, baroreflex sensitivity and oxidative stress. ${ }^{19,20} \mathrm{Fi}$ nally, studies on the effects of regular endurance exercise on mood 
state in women during the menopausal transition, demonstrate significant improvements in depressive symptoms and reduction in menopause-related anxiety and mood disorders after endurance exercise training., ${ }^{4,21}$

Despite the accumulated evidence on the effects of endurance exercise on the postmenopausal years, there is a scarcity of data on the effects of exercise on the critical time period of the perimenopausal years. The limited studies conducted demonstrate the promising effects of endurance exercise training on the health and exercise capacity of perimenopausal women but also stress the need for more studies to be conducted in this understudied population. ${ }^{11,22-25}$ In the few studies that have been conducted, it is evident that moderate-intensity endurance exercise primarily in the form of walking can induce significant improvements in body weight, body fat, aerobic fitness and overall exercise capacity in perimenopausal women. ${ }^{11,23}$ Moreover, endurance exercise has been shown to induce significant improvements in independent cardiovascular risk factors such as glucose and lipid levels and overall quality of life in pre and perimenopausal women..$^{22,24,25}$ However, no research studies have investigated the effects of exercise on the mood and cognitive state of perimenopausal women, leaving a gap in the literature on a crucial time period for women's mental health. There is an urgent need to conduct more research studies on the perimenopausal years in order to identify optimal lifestyle treatment plans that can prevent the development of mental and non-mental health problems at an early stage.

High-intensity interval exercise (HIIE) has recently emerged as a favourable exercise modality for clinical populations with obesity, type 2 diabetes and cardiovascular disease leading to comparable or even faster improvements in exercise capacity, body composition and cardiometabolic health parameters. ${ }^{26-29}$ Specifically, similar or faster improvements in exercise capacity, insulin resistance, blood pressure and cardiac function have been documented in older clinical populations with metabolic syndrome, cardiovascular disease and type 2 diabetes when training with HIIE compared to continuous exercise. ${ }^{29,30}$ In older male and female obese populations, HIIE has been documented to have similar effectiveness with moderate intensity continuous exercise across all body composition measures but in a more time-efficient way, by requiring less than $40 \%$ training time compared to continuous exercise. ${ }^{27}$ Furthermore, HIIE has been reported to lead to similar enjoyment and exercise adherence levels in overweight and obese older males and females with continuous exercise, demonstrating the important role that it might have on exercise compliance in this population. ${ }^{31}$ However, limited evidence exists on the effects of HIIE on the exercise capacity and health on the specific high-risk time periods of peri and menopause. In postmenopausal women, 16-weeks of HIIE have been shown to lead to greater weight loss and improvements in body composition compared to continuous moderate intensity exercise in healthy postmenopausal women ${ }^{32}$ and greater reductions in intra-abdominal adiposity in obese postmenopausal type 2 diabetic women. ${ }^{12,33,34}$ Moreover, Mandrup et $\mathrm{al}^{12}$ has demonstrated significant improvements in body composition as observed by increases in lean body mass and reductions in fat mass and improvements in cardiometabolic risk factors in postmenopausal women after HIIE training. Finally, Egelund et $\mathrm{al}^{35}$ studied the effects of HIIE on early postmenopausal women and found marked improvements in cardiac function in terms of systolic and diastolic function after 12 weeks of cycle interval training.

The effects of HIIE on the perimenopausal years is largely understudied. In younger overweight and obese women, HIIE has been shown to be a potentially more effective mode of exercise compared to continuous exercise, in terms of time-efficiency and perception of effort while significantly improving aerobic fitness, blood glucose and lipid levels. ${ }^{36}$ In a recent study that our research group conducted investigating the effects of an acute bout of interval exercise on psychophysiological adaptations in overweight perimenopausal women, we found that one acute bout of HIIE alone was effective in inducing significant improvements in the systolic and diastolic blood pressure of this population. ${ }^{37}$ Moreover, it led to improvements in mood, exercise enjoyment and exercise tolerance, especially when combined with high carbohydrate versus high protein pre-exercise feedings, while no changes were noted in cognitive capacity. ${ }^{37}$ Further, in unpublished preliminary data from our research group, we have found that one bout of interval exercise can induce similar fat oxidation rates and exercise enjoyment compared to continuous exercise in a group of overweight perimenopausal women, mimicking the findings of an earlier study in younger women where 7 sessions of HIIE over a two-week period resulted in marked elevations in whole body and skeletal muscle fatty acid oxidation. ${ }^{38}$ It is important to note that the improvements seen in our group of perimenopausal women with HIIE were achieved in approximately half the exercise duration compared to continuous exercise, suggesting significant practical training applications for this population, given that "lack of time" is the most commonly cited barrier to regular exercise participation. Research on the effects of long-term HIIE have also shed some light on the potential advantageous effects of this mode of exercise for older women. Seidelin et $\mathrm{al}^{39}$ recently demonstrated improvements in aerobic capacity in late pre-menopausal women after intermittent exercise training for 12 weeks. Moreover, the group of Mandrup et $\mathrm{al}^{12}$ reported that a 3-month high-intensity aerobic training intervention induced similar levels of improvements in cardiovascular disease and type 2 diabetes risk factors in pre-menopausal women. Finally, Egelund et $\mathrm{al}^{35}$ showed that a 12 -week HIIE intervention on pre-menopausal women led to significant positive adaptations in cardiac function such as left ventricle mass and left atrial enddiastolic and end-systolic volumes. ${ }^{40}$ More evidence is required to further investigate and establish the effectiveness of HIIE on the perimenopausal years, especially in terms of neurological and cognitive health disturbances in this population. ${ }^{41}$

It is evident that additional investigations are needed to assess the effects of exercise on the critical perimenopausal years. Perimenopause offers the unique opportunity for chronic disease risk reduction in women at an early stage, prior to menopause, at which time point the development of chronic diseases is accentuated. ${ }^{42}$ More studies need to be conducted on clinical exercise modalities such as high-intensity interval exercise clinical proto- 
cols that can optimally counteract the effects of menopause on women's health and reduce the risk of developing mental healthrelated comorbidities. Re-evaluating the optimal exercise mode for this population could lead to a more effective exercise prescription that could enhance exercise adherence and enjoyment while allowing women to exercise in a more time-efficient manner compared to the traditionally prescribed exercise programs.

\section{REFERENCES}

1. Bacon JL. The menopausal transition. Obstet Gynecol Clin North Am. 2017; 44(2): 285-296. doi: 10.1016/j.ogc.2017.02.008

2. Hale GE, Robertson DM, Burger HG. The perimenopausal woman: endocrinology and management. J Steroid Biochem Mol Biol. 2014; 142: 121-131. doi: 10.1016/j.jsbmb.2013.08.015

3. Van Dijk GM, Kavousi M, Troup J, Franco OH. Health issues for menopausal women: the top 11 conditions have common solutions. Maturitas. 2015; 80(1): 24-30. doi: 10.1016/j.maturitas.2014.09.013

4. Bromberger JT, Schott LL, Kravitz HM, et al. Longitudinal change in reproductive hormones and depressive symptoms across the menopausal transition: Results from the Study of Women's Health Across the Nation (SWAN). Arch Gen Psychiatry. 2010; 67: 598-607. doi: 10.1001/archgenpsychiatry.2010.55

5. De Kruif M, Spijker AT, Molendijk ML. Depression during the perimenopause: A meta-analysis. J Affect Disord. 2016; 206: 174180. doi: $10.1016 /$ j.jad.2016.07.040

6. Gibbs Z, Lee S, Kulkarni J. Factors associated with depression during the perimenopausal transition. Womens Health Issues. 2013; 23(5): e301-e307. doi: 10.1016/j.whi.2013.07.001

7. Brinton RD, Yao J, Yin F, Mack WJ, Cadenas E. Perimenopause as a neurological transition state. Nat Rev Endocrinol. 2015; 11(7): 393-405. doi: 10.1038/nrendo.2015.82

8. Georgakis MK, Kalogirou EI, Diamantaras AA, et al. Age at menopause and duration of reproductive period in association with dementia and cognitive function: A systematic review and meta-analysis. Psychoneuroendocrinology. 2016; 73: 224-243. doi: 10.1016/j.psyneuen.2016.08.003

9. Wariso BA, Guerrieri GM, Thompson K, et al. Depression during the menopause transition: Impact on quality of life, social adjustment, and disability. Arch Womens Ment Health. 2017; 20(2): 273 282. doi: 10.1007/s00737-016-0701-x

10. Kemmler W, Kohl M, von Stengel S. Long-term effects of exercise in postmenopausalwomen: 16-year results of the erlangen fitness and osteoporosis prevention study (EFOPS). Menopause. 2017; 24(1): 45-51. doi: 10.1097/GME.0000000000000720
11. Gao HL, Gao HX, Sun FM, Zhang L. Effects of walking on body composition in perimenopausal and postmenopausal women: a systematic review and meta-analysis. Menopause. 2016; 23(8): 928-934. doi: 10.1097/GME.0000000000000627

12. Mandrup CM, Egelund J, Nyberg M. Effects of high-intensity training on cardiovascular risk factors in pre-menopausal and postmenopausal women. Am J Obstet Gynecol. 2017; 216(4): 384.e1-384. e11. doi: 10.1016/j.ajog.2016.12.017

13. Mendoza N, De Teresa C, Cano A, et al. Benefits of physical exercise in postmenopausal women. Maturitas. 2016; 93: 83-88. doi: 10.1016/j.maturitas.2016.04.017

14. Ross R, Janssen I. Physical activity, total and regional obesity: Dose-response considerations. Med Sci Sports Exerc. 2001; 33(6 Suppl): S521-S527. doi: 10.1097/00005768-200106001-00023

15. Bueno-Notivol J, Calvo-Latorre J, Alonso-Ventura V, et al. Effect of programmed exercise on insulin sensitivity in postmenopausal women: A systematic review and meta-analysis of randomized controlled trials. Menopause. 2017; 24(12): 1404-1413. doi: 10.1097/GME.0000000000000936

16. Cuff DJ, Meneilly GS, Martin A, Ignaszewski A, Tildesley HD, Frohlich JJ. Effective exercise modality to reduce insulin resistance in women with type 2 diabetes. Diabetes Care. 2003; 26(11): $2977-$ 2982. doi: $10.2337 /$ diacare.26.11.2977

17. Giannopoulou I, Ploutz-Snyder LL, Carhart R, et al. Exercise is required for visceral fat loss in postmenopausalwomen with type 2 diabetes. J Clin Endocrinol Metab. 2005; 90(3): 1511-1518. doi: $10.1210 /$ jc. $2004-1782$

18. Giannopoulou I, Fernhall B, Carhart R, et al Effects of diet and/or exercise on the adipocytokine and inflammatory cytokine levels of postmenopausal women with type 2 diabetes. Metabolism. 2005; 54(7): 866-875. doi: 10.1016/j.metabol.2005.01.033

19. Jarrete AP, Novais IP, Nunes HA, Puga GM, Delbin MA, Zanesco A. Influence of aerobic exercise training on cardiovascular and endocrine-inflammatory biomarkers in hypertensive postmenopausal women. J Clin Transl Endocrinol. 2014; 1: 108-114. doi: 10.1016/j.jcte.2014.07.004

20. Lin YY, Lee SD. Cardiovascular benefits of exercise training in postmenopausal hypertension. Int J Mol Sci. 2018; 19(9). pii: E2523. doi: $10.3390 /$ ijms19092523

21. Sternfeld B, Dugan S. Physical activity and health during the menopausal transition. Obstet Gynecol Clin North Am. 2011; 38(3): 537-566. doi: 10.1016/j.ogc.2011.05.008

22. Coll-Risco I, Borges-Cosic M, Acosta-Manzano P, Camiletti-Moirón D, Aranda P, Aparicio VA. Effects of concurrent exercise on cardiometabolic status during perimenopause: the 
FLAMENCO Project. Climacteric. 2018; 21(6): 559-565. doi: 10.1080/13697137.2018.1526892

23. Ring-Dimitriou S, Steinbacher P, von Duvillard SP, Kaessmann H, Müller E, Sänger AM. Exercise modality and physical fitness in perimenopausal women. Eur J Appl Physiol. 2009; 105(5): 739-747. doi: 10.1007/s00421-008-0956-7

24. Zhang J, Chen G, Lu W, et al. Effects of physical exercise on health-related quality of life and blood lipids in perimenopausal women: A randomized placebo-controlled trial. Menopause. 2014; 21(12): 1269-1276. doi: 10.1097/GME.0000000000000264

25. Wu L, Chen R, Ma D, Zhang S, Walton-Moss B, He Z. Effects of lifestyle intervention improve cardiovascular disease risk factors in community-based menopausal transition and early postmenopausal women in China. Menopause. 2014; 21(12): 1263-1268. doi: 10.1097/GME.0000000000000248

26. Hollekim-Strand SM, Bjørgaas MR, Albrektsen G, Tjønna AE, Wisløff U, Ingul CB. High-intensity intervalexercise effectively improves cardiac function in patients with type 2 diabetes mellitus and diastolic dysfunction: A randomized controlled trial. J Am Coll Cardiol. 2014; 64(16): 1758-1760. doi: 10.1016/j.jacc.2014.07.971

27. Keating SE, Johnson NA, Mielke GI, Coombes JS. A systematic review and meta-analysis of interval training versus moderateintensity continuous training on body adiposity. Obes Rev. 2017; 18(8): 943-964. doi: 10.1111/obr.12536

28. Su L, Fu J, Sun S, et al. Effects of HIIT and MICT on cardiovascular risk factors in adults with overweight and/or obesity: A meta-analysis. PLoS One. 2019; 14(1): e0210644. doi: 10.1371/ journal.pone.0210644

29. Wisløff U, Ellingsen O, Kemi OJ. High-intensity interval training to maximize cardiac benefits of exercise training? Exerc Sport Sci Rev. 2009; 37(3): 139-146. doi: 10.1097/JES.0b013e3181aa65fc

30. Weston KS, Wisløff U, CoombesJS. High-intensity interval training in patients with lifestyle-induced cardiometabolic disease: a systematic review and meta-analysis. Br J Sports Med. 2014; 48(16): 1227-1234. doi: 10.1136/bjsports-2013-092576

31. Vella CA, Taylor K, Drummer D. High-intensity interval and moderate-intensity continuous training elicit similar enjoyment and adherence levels in overweight and obese adults. Eur J Sport Sci. 2017; 17(9): 1203-1211. doi: 10.1080/17461391.2017.1359679

32. Grossman JA, Arigo D, Bachman JL. Meaningful weight loss in obese postmenopausal women: a pilot study of high-intensity interval training and wearable technology. Menopause. 2018; 25(4): 465-470. doi: 10.1097/GME.0000000000001013
33. Maillard F, Rousset S, Pereira B, et al. High-intensity interval training reduces abdominal fat mass in postmenopausal women with type 2 diabetes. Diabetes Metab. 2016; 42(6): 433-441. doi: 10.1016/j.diabet.2016.07.031

34. Maillard F, Pereira B, Boisseau N. Effect of high-intensity interval training on total, abdominal and visceral fat mass: A metaanalysis. Sports Med. 2018; 48(2): 269-288. doi: 10.1007/s40279017-0807-y

35. Egelund J, Jørgensen PG, Mandrup CM, et al. Cardiac adaptations to high-intensity aerobic training in pre-menopausal and recent postmenopausal women: The copenhagen women study. J Am Heart Assoc. 2017; 6(8). pii: e005469. doi: 10.1161/ JAHA.117.005469

36. Kong Z, Sun S, Liu M, Shi Q. Short-term high-intensity interval training on body composition and blood glucose in overweight and obese young women. J Diabetes Res. 2016; 2016: 4073618. doi: $10.1155 / 2016 / 4073618$

37. Kotopoulea-Nikolaidi M, Watkins E, Giannopoulou I. Effects of high carbohydrate vs. high protein pre-exercise feedings on psychophysiological responses to high intensity interval exercise in overweight perimenopausal women. Front Nutr. 2019; 5: 141. doi: 10.3389/fnut.2018.00141

38. Talanian JL, Galloway SD, Heigenhauser GJ, Bonen A, Spriet LL. Two weeks of high-intensity aerobic interval training increases the capacity for fat oxidation during exercise in women. $J$ Appl Physiol (1985). 2007; 102(4): 1439-1447. doi: 10.1152/japplphysiol.01098.2006

39. Seidelin K, Nyberg M, Piil P, Jørgensen NR, Hellsten Y, Bangsbo J. Adaptations with intermittent exercise training in post- and pre-menopausal women. Med Sci Sports Exerc. 2017; 49(1): 96-105. doi: 10.1249/MSS.0000000000001071

40. Miszko TA, Cress ME. A lifetime of fitness: Exercise in the perimenopausal and postmenopausal woman. Clin Sports Med. 2000; 19(2): 215-232. doi: 10.1016/S0278-5919(05)70200-3

41. Panagiotakos D, Pitsavos C, Chrysohoou C, Skoumas J, Stefanadis C. Status and management of blood lipids in Greek adults and their socio-demographic, life-style and dietary factors: The ATTICA study. Blood lipid distribution in Greece. Atherosclerosis. 2004; 173(2): 351-361. doi: 10.1016/j.atherosclerosis.2003.12.03

42. Wisløff U, Støylen A, Loennechen JP, et al. Superior cardiovascular effect of aerobic interval training versus moderate continuous training in heart failure patients: A randomized study. Circulation. 2007; 115: 3086-3094. doi: 10.1161/CIRCULATIONAHA.106.675041 